\title{
XEOM 1 - A novel microscopy system for the chemical imaging of heritage metal surfaces
}

\author{
Mark Dowsett ${ }^{1{ }^{*}}$, Matt Hand ${ }^{2}$, Pieter-Jan Sabbe ${ }^{3 \dagger}$, Paul Thompson ${ }^{4+}$ and Annemie Adriaens ${ }^{3+}$
}

\begin{abstract}
Background: We describe a novel microscopy system which can obtain chemical maps from the surfaces of heritage metals in air or a controlled environment. The microscope, $\mathrm{x}$-ray excited optical microscope Mk 1 (XEOM 1), forms images from $x$-ray excited optical luminescence (XEOL) induced by illuminating a few square millimetres of the sample with monochromated $x$-rays (broad beam or macroprobe illumination). XEOL is a spectroscopy tool in its own right and can, under the right circumstances, also be a vehicle for $x$-ray absorption spectroscopy. This (usually) synchrotron based technique provides information on the chemical state and short-range atomic order of the top few microns of a surface. It is thus well suited to heritage metal corrosion studies and is complementary to synchrotron x-ray diffraction.

Results: Imaging can be performed by scanning the sample under an x-ray microprobe. We show elsewhere that the power density needed for image acquisition on a reasonable time-scale is high enough to damage a patina and modify its chemistry. Although the damaged region may be invisible to the human eye, the data are characteristic of the damage and not the native chemistry of the surface. A macrobeam power density can be 4 orders of magnitude smaller than that for a microbeam and no surface modification was observed on test samples. Features of the instrument are demonstrated using copper test surfaces with a spatially varying patination to establish the ground work for the imaging of copper, cuprite, nantokite and atacamite/paratacamite and a first application from a bronze chain mail link. In parallel we have developed a suite of imaging software which can process XEOM image stacks to produce reduced data sets characteristic of various aspects of the surface chemical map. These include edge-shift (oxidation state) images and edge height (high contrast) images and spectra from user defined regions of interest.

Conclusions: The technique can map the oxidation state of a surface from shifts in the absorption edge energy across columns of pixels in an image set, and map particular compounds from their characteristic XANES spectra. Optically filtered images give improved chemical selectivity and the data sets contain as yet untapped information sources.
\end{abstract}

Keywords: Patina Mapping, X-Ray excited optical luminescence (XEOL), XEOL-XANES

\section{Background}

One effect of the bombardment of material surfaces with $\mathrm{x}$-rays is the emission of much lower energy electromagnetic radiation in the visible and near-visible bands. (herein referred to as trans-visible) [1-4]. This phenomenon, $x$-ray excited optical luminescence (XEOL), is the basis of a spectroscopy tool in its own right but, since the intensity of the emission is dependent on the probability of absorption of the $\mathrm{x}$-rays onto core levels, it can also carry the information for $\mathrm{x}$-ray absorption near edge spectroscopy (XANES)

\footnotetext{
*Correspondence: markdowsett.esa@gmail.com

${ }^{\dagger}$ Equal contributors

'Department of Physics, University of Warwick, Coventry CV4 7AL, UK

Full list of author information is available at the end of the article
}

and extended $\mathrm{x}$-ray absorption fine structure (EXAFS) [5-9]: This is because the trans-visible light will contain a contribution from particular radiative transitions excited downstream from the core-level photoexcitation, and therefore modulated in the same way. (However, this contribution must be sufficiently intense to compete with the emission arising from the non resonant excitation of states below the core level in question or it will not be discernible. This places limitations both on the minimum detectable concentration of the species whose core level is being probed, and the maximum concentration of emitters responsible for non-resonant background.) Thus, in favourable cases, by scanning the energy of the incident $\mathrm{x}$-rays across the absorption 
energy of a particular core level (e.g. copper K- $\alpha$, lead LIII) and beyond and measuring the intensity of the transvisible emission, information on short range order, oxidation state, chemical species, etc. can be obtained. The resulting spectroscopies are known collectively as optically detected $\mathrm{x}$-ray absorption spectroscopy (ODXAS) and individually as XEOL-XANES and XEOL-EXAFS. Elsewhere $[8,10]$ we summarize some differences in the appearance of signals from XANES and XEOL-XANES. We also show that they easily detect far thinner layers of surface corrosion on heritage metals compared to $\mathrm{x}$-ray fluorescence, the conventional way to measure XAS data from surfaces which has an information depth of several micrometres. In general, ODXAS is likely to be surface specific to around the skin depth at the optical frequency observed: i.e. $100-200 \mathrm{~nm}$ in good conductors, but potentially more in insulators. It therefore lies between conventional XAS and total electron yield XAS (TEYXAS) [11] in its surface specificity. The emission from metals and their corrosion products is broadband, typically covering the wavelength range from $<250 \mathrm{~nm}$ to $>1 \mu \mathrm{m}$. However, many different end-states contribute across this band so that emission from particular transitions may be studied by using colour filters, giving further selectivity.

In general, for any analytical method, chemical and structural imaging can be done in one of two ways: A fine probe (a micro or nanoprobe) of the incident radiation may be scanned across the sample (or where the probe is difficult to scan, the sample may be moved across the probe) $[7,12-14]$. The emission is recorded as a function of probe position. An advantage of this method is that different information channels can be collected from the same point on the sample using parallel detection [9]. Alternatively, a relatively large area of sample may be illuminated simultaneously with a broad beam (a macroprobe), and the emission imaged onto a detector with lateral resolution, e.g. a pixelated detector such as a charge coupled device (CCD) [6,14-18], sometimes with energy resolution on each pixel $[19,20]$. This is just applying the principle of the microscope. An advantage of this method is that movement of the beam, due, for example to monochromator scanning, does not shift the image. However, achieving uniform $\mathrm{x}$-ray illumination over the imaged area may not be straightforward because the beam from a synchrotron source often contains internal structure.

Both approaches will result in dead-time (in the sense of time when data cannot be taken). For example, in both the scanning of the monochromator and the mechanical rastering of a sample no data can be taken until the position has stabilized. With full field imaging, the read out speed of a CCD must be moderated to reduce noise in the images. These effects must be considered when designing experiments to minimize the elapsed time, and the overall dose accumulated in the sample.

Both the microprobe and the macroprobe are ubiquitous in electron microscopy, ion imaging techniques, $\mathrm{x}$-ray methods, optical spectroscopy and so on. (Near-field optical techniques may also be used [21] but these have similar beam density implications to microprobes.) The lateral resolution of a microprobe is ultimately limited either by the probe size, or by the dimensions of the interaction volume. For the macroprobe, the ultimate resolution is determined by aberrations and diffraction in the image-forming device or by limitations such as pixel size and cross talk between pixels in the detector. (Practical lateral resolution is often determined by statistical fluctuation in either case [22].) The microprobe method usually offers the highest lateral resolution but the microscope method may be orders of magnitude faster in image collection since each part of the image is simultaneously acquired. Moreover, to achieve practical collection times, the power density in the microprobe must be orders of magnitude higher than in the macroprobe (since the same total dose must be delivered to a pixel for the same statistical precision).

As we show elsewhere $[8,10]$, the $\mathrm{x}$-ray power density used in a microprobe for XAS and ODXAS imaging alters copper and bronze corrosion products on a timescale from milliseconds to tens of seconds through a variety of processes. Such images are therefore unlikely to be representative of the original surface. For example [10], we have shown that using a microprobe with a power density of $60 \mathrm{~W} \mathrm{~mm}^{-2}$, a thin nantokite coating (observed in the ODXAS channel, but too thin to be seen in conventional XAS) is progressively hydrolysed to paratacamite in the beam over a period of seconds to minutes. In contrast, in a macroprobe of $20 \mu \mathrm{W} \mathrm{mm}{ }^{-2}$ (typical of a bending magnet) the ODXAS observed from the thin patina and a bulk reference of $\mathrm{CuCl}$ are stable over time and identical [8]. Effects analogous to the bleaching seen in conventional fluorescence microscopy [23] are also seen in our microprobe data [10]. Since optical components and pixelated light detectors are widely available and relatively inexpensive, imaging using XEOL provides an attractive alternative for collecting XAS images using a macroprobe. We therefore developed a broadband microscopy system (XEOM 1) [16] which will transfer XEOL from an area of $4 \mathrm{~mm}^{2}$ to a broadband CCD detector. XEOM 1 allows the sample to be in air, in a controlled atmosphere, or even immersed in an electrochemical cell.

\section{Results \\ XEOM data structure}

A typical XANES spectrum contains 100 or more points. XEOM 1 will acquire one image at each energy in the 
scan, and the data set will therefore consist of 50 or more images. This is known as an image stack and is shown schematically in Figure 1. Flat field and dark field corrections (as is usual for CCD images) are applied to each image in the stack and the images are then normalized to the beam monitor (usually an ion chamber) to remove effects due to changes in beam flux over time (a typical correction to synchrotron data). Localized effects due to beam structure are not corrected for at present; instead care is taken to get the illumination as uniform as possible by using a beam which is not too tightly focussed. Beam shaping methods [24] may be attempted in future. Each column of pixels in the stack contains its own XANES spectrum, characteristic of the small region of sample (virtual pixel) imaged onto the corresponding detector pixel. Adjacent pixels may be added together (binned) to improve the statistics at the expense of lateral resolution, or pixels in a userdefined region of interest (RoI) might be combined to get the spectrum from a patch of corrosion (See e.g. RoI 1 and RoI 2 in Figure 1). Ideally, XANES spectra would be acquired at least over a range from $50 \mathrm{eV}$ before to $100 \mathrm{eV}$ after the edge depending on the nature of the pre-edge signal, characteristic post-edge spectral features, and whether linear combinational analysis was to be attempted. For the image stacks presented here, the range was more restricted $(20 \mathrm{eV}$ pre-edge and $20-25 \mathrm{eV}$ post-edge) in order to reduce the stack acquisition time. This limited range is justified below. With current and foreseeable technology, the acquisition of ODXAS at low enough statistical fluctuation for linear combination analysis across $300 \mathrm{eV}$ of spectrum at $1 \mu \mathrm{m}$ lateral resolution is likely to take several days per image stack. Clearly the challenge is to find other ways of using the data as shown in what follows.

\section{XEOM 1 data from cuprite and nantokite test samples}

The XEOL from different compounds generally has a different colour balance or may even show up in a narrow band. Chemical information can therefore be obtained by imaging through colour filters in addition to using $x$-ray spectroscopy [25]. Figure $2(a, b)$ shows images of a copper grid coated with nantokite resting on a copper coupon with an oxidised surface. The oxide is predominantly cuprite $(>90 \%)$ with some tenorite as an impurity. The images are taken from the stack at $9 \mathrm{keV}$, just past the k-edge. The energy range in the stack is sufficient to distinguish between XANES of copper, cuprite, nantokite, and copper hydroxychlorides (in the event that the nantokite had hydrolysed). Figure 2(a) is an image through a blue fluorescence line filter and favours emission from the coupon surface. Conversely, the image through a red fluorescence line filter (Figure 2(b)) shows more intense emission from the mesh. Figure 2(c) shows XEOL-XANES spectra from RoIs on the mesh

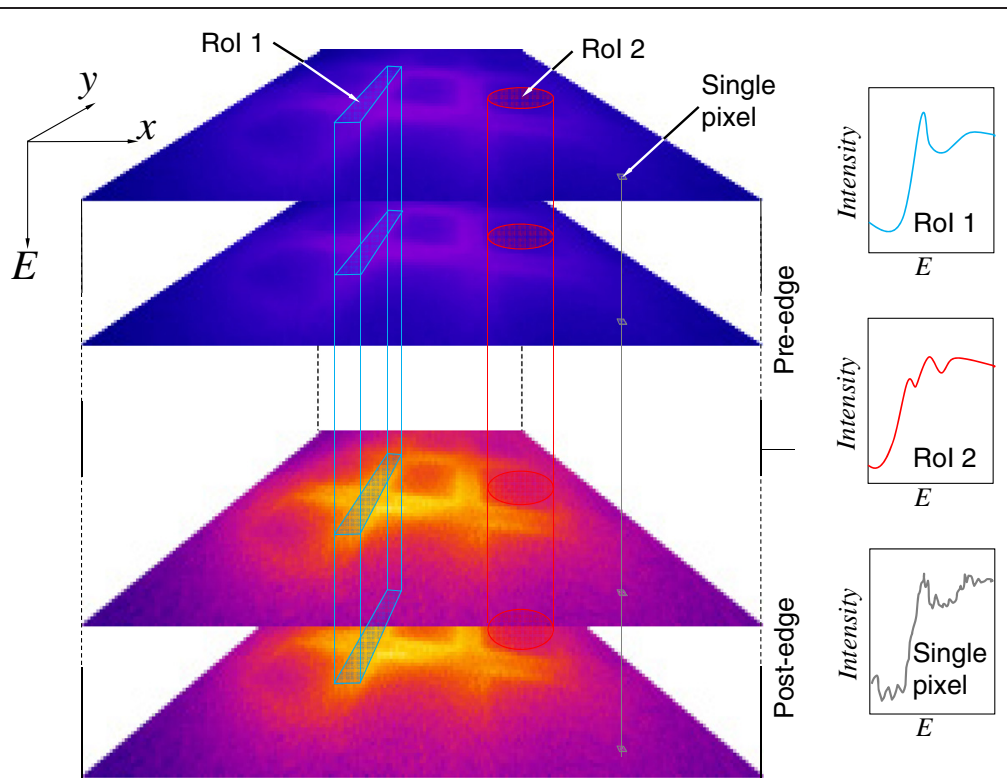

Figure 1 The data structure for $\mathbf{x}$-ray excited optical luminescence microscopy. The energy of the broad incoming x-ray beam is stepped across an absorption edge of the chemical element under investigation in, typically 100 or more small increments. An optical luminescence image is acquired for each energy in the scan. The images form a stack which can be processed with suitable software to extract XEOL-XANES data from a column of single pixels (grey delineation and spectrum) or from user-defined regions of interest (Rol) (red and blue delineation and spectra). The power density in the $x$-ray beam is $<7.2 \times 10^{-3} \mathrm{~W} \mathrm{~mm}^{-2}$. 

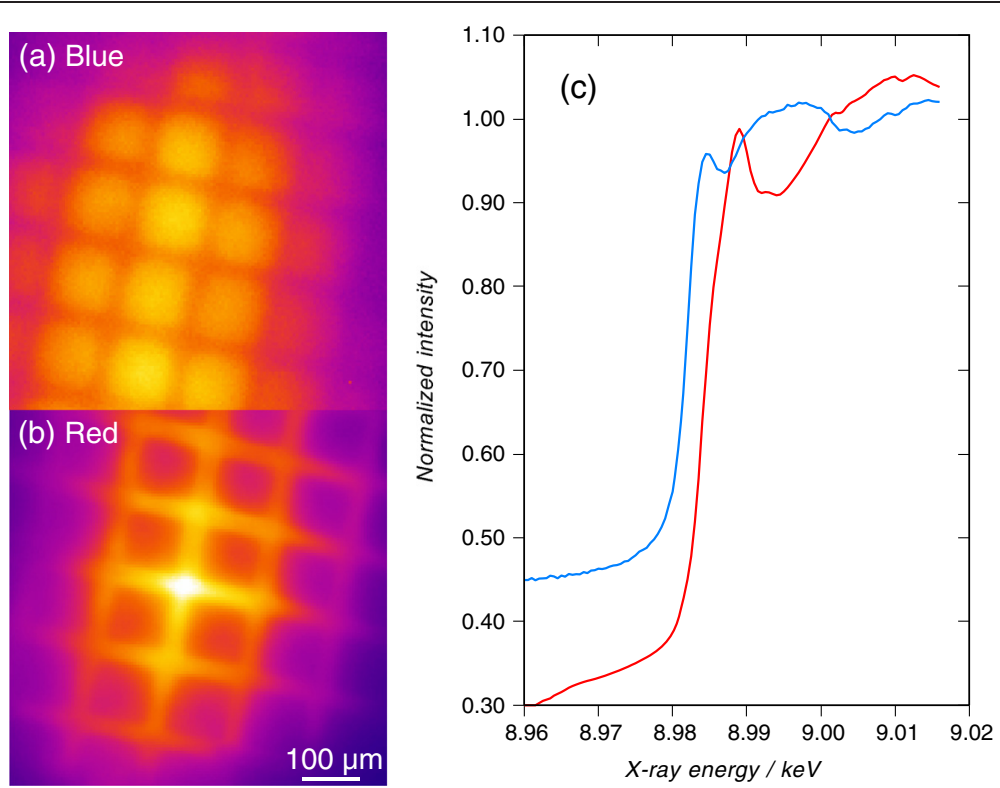

Figure 2 XEOM Cu-K edge data from a nantokite coated mesh on a cuprite coated coupon. (a) Post edge image from a stack acquired through a blue fluorescence line filter (a similar image is seen through green and UV filters). The emission from the mesh bars (nantokite) is lower at short wavelengths that that from the holes through which the coupon is visible (cuprite). (b) Image from a stack using a red fluorescence line filter. The emission from the mesh is now stronger than that from the coupon. (c) XEOL-XANES data from Rols in the holes in the blue image stack (blue curve) and from the bars of the red image stack (red curve). The spectra are characteristic, respectively, of cuprite and nantokite. Note the edge shift to higher energy between the $\mathrm{Cu}(\mathrm{I})$ and $\mathrm{Cu}(\mathrm{II})$ states:

holes in Figure 2(a) (blue curve, characteristic of cuprite) and mesh bars in Figure 2(b). (red curve, characteristic of nantokite).

\section{A bronze chain mail link from the Mary Rose}

Many different types of information may be extracted from an image stack. Figure 3 shows data obtained from a bronze chain mail link recovered from the wreck of King Henry VIII's flagship the Mary Rose (Item MR81A1436). The link is in the form of a loop $\varnothing 9.1 \mathrm{~mm}$ made from bronze wire $\varnothing 1.2 \mathrm{~mm}$. It has been subjected to conservation treatment to remove chlorides absorbed during 437 years of immersion in the sea. The data are abstracted from image stacks across the $\mathrm{Cu}-\mathrm{K}$ absorption edge whose energy range was chosen to be sufficient for the purpose of mapping the energy shift of the edge. Figure 3(a) is a photograph of the link showing its mounting and the analysed area. Figure 3(b) shows a XEOL image (ironbow colour scale) of a region of the link surface $2 \times 0.5 \mathrm{~mm}^{2}$ formed by subtracting an image taken with incident $\mathrm{X}$-rays at $8.96 \mathrm{keV}$ (just before the copper K-edge) from one taken at $9.016 \mathrm{keV}$ (just after the K-edge). This removes background fluorescence from the image and heightens the contrast (and may in some circumstances provide elemental contrast). The changes in brightness across the surface show that it is inhomogeneous in composition but do not necessarily provide more information as the presence of surface contaminants (e.g. sodium salts) fluorescing in sympathy with the $\mathrm{Cu}-\mathrm{K}$ related processes cannot be ruled out. It is well known that the position (in energy) of the absorption edge is sensitive to the oxidation state of the element. So, if the edge position is found for each column of pixels, and associated with a false colour scale one obtains an oxidation state map (Figure 3(c)). On average there is an approximately $5 \mathrm{eV}$ shift in edge energy from the green regions to the blue. These regions do not correlate exactly with the bright regions in Figure 3(a) and suggest the presence of $\mathrm{Cu}$ (I) (light green regions) and $\mathrm{Cu}$ (II) (blue regions) compounds. XEOL-XANES spectra were extracted from the image stack using the two RoIs depicted in Figure 3(b) in green (split into two parts and sampling $\mathrm{Cu}(\mathrm{I})$ regions) and blue (sampling a $\mathrm{Cu}(\mathrm{II})$ region). These appear in Figure 4 where a XEOL-XANES spectrum from a pure copper coupon is added for comparison. The shape of the spectrum from RoI 1 is consistent with cuprite $\left(\mathrm{Cu}_{2} \mathrm{O}\right)$, whilst the edge shift in the data from RoI 2 suggests the presence of tenorite $(\mathrm{CuO})$. Both spectra, however, retain some features of copper which suggests that the layers are $200 \mathrm{~nm}$ or less thick or patchy on the microscale. The reason for the pre-edge spike (arrowed) is not known.

\section{Conclusions}

Imaging of copper patinas using high intensity X-ray microprobes can change the composition of the surface thereby invalidating the results. A similar effect would 


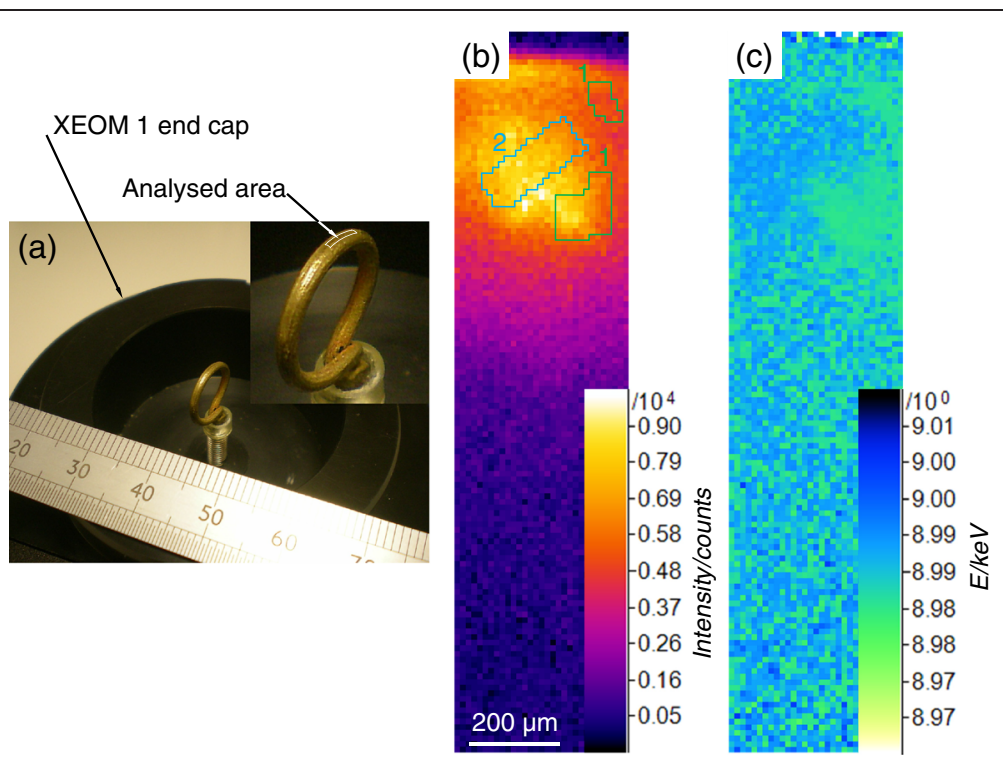

Figure 3 Cu-K edge XEOL-XANES imaging of a bronze chain mail link recovered from the Mary Rose (item MR81A1436). (a) The link mounted in the end cap of XEOM 1. Inset shows the approximate analysed area. (b) XEOL image (ironbow colour scale) of the surface showing some inhomogeneity. The image was formed by subtracting a pre-edge image from a post edge one to remove the fluorescence background and heighten the contrast. The brighter regions may correspond to a thicker patina, or to resonant photon emission from other impurities such as sodium salts. (c) The Cu-K edge position in each pixel column from the corresponding image stack converted to an image give information on the local oxidation state. The green areas correspond to $\mathrm{Cu}(\mathrm{I})$ whilst the blue with an average shift to higher energy around $5 \mathrm{eV}$ correspond to $\mathrm{Cu}(\mathrm{II})$.

be expected for other surfaces, especially when $\mathrm{x}$-ray energies close to absorption edges of the material are used, and irrespective of the actual technique in use e.g. XAS, synchrotron $\mathrm{X}$-ray diffraction SR-XRD, XEOL and other photoluminescence-based techniques. It should never be assumed that the input flux from a synchrotron is nondestructive; this has to be demonstrated for any particular material system. By using a large area beam, with a power density several orders of magnitude lower, XEOL microscopy has the potential to provide similar information in a less destructive or non-destructive way.

The early results from XEOM 1 presented here show chemical speciation of copper compounds on test structures and a heritage metal surface. Spectra extracted from columns of pixels in image stacks give chemical speciation with lateral resolution in the range 10 micrometres. Speciation can be improved by taking images through colour filters.

\section{Methods}

\section{Construction of XEOM 1}

The microscope is described in more detail elsewhere [16] but an outline is given here. With reference to Figure 5: The microscope is constructed mainly from black acetal copolymer stiffened by mounting on an aluminium optical rail. The acetal is light, impact proof, non-reflective, inexpensive, easy to machine to high tolerances, and we find experimentally that it has very low fluorescence in the visible when irradiated with $x$-rays. The body of the microscope consists of three main parts: (i) A light tight sample housing with a varied selection of stubs and mountings and ports at $10^{\circ}, 45^{\circ}$ and $60^{\circ}$ for admitting $x$-rays, sample illumination systems, sample viewing cameras and supplementary detectors. The end of the housing may be removed for the insertion of a custom electrochemical/ environmental cell [26]. (ii) An optics housing containing the lenses, optical filters, and a remote controlled focussing mechanism. The last stage of this housing is lined with $0.5 \mathrm{~mm}$ thick lead foil to prevent $\mathrm{x}$-rays penetrating the plastic wall and reaching the CCD camera. (iii) A CCD camera with a Peltier cooled broadband sensor. The first camera employed was an FLI Microline ML1109 (Finger Lakes Instrumentation Inc. USA) fitted with a Hamamatsu S10140-1009 sensor (Hamamatsu Photonics K. K., Japan). The sensor is $24.6 \times 6 \mathrm{~mm}^{2}$ in size with $1024 \times 506$ active pixels which with the magnification of the XEOM 1 optics $(12 \times)$ gave an imaged area of $2 \times 1 \mathrm{~mm}^{2}$ at the sample. This device was used to gather the data in Figures 3 and 4. Problems with the sensor led to the replacement of the camera with an Andor Ikon-L 936 (Andor Technology Ltd. Northern Ireland) fitted with an e2V CCD42-40 NIMMO sensor (e2V Technologies Ltd., UK). The sensor is $27.6 \times$ $27.6 \mathrm{~mm}^{2}$ with $2048 \times 2048$ pixels. This gives an imaged 


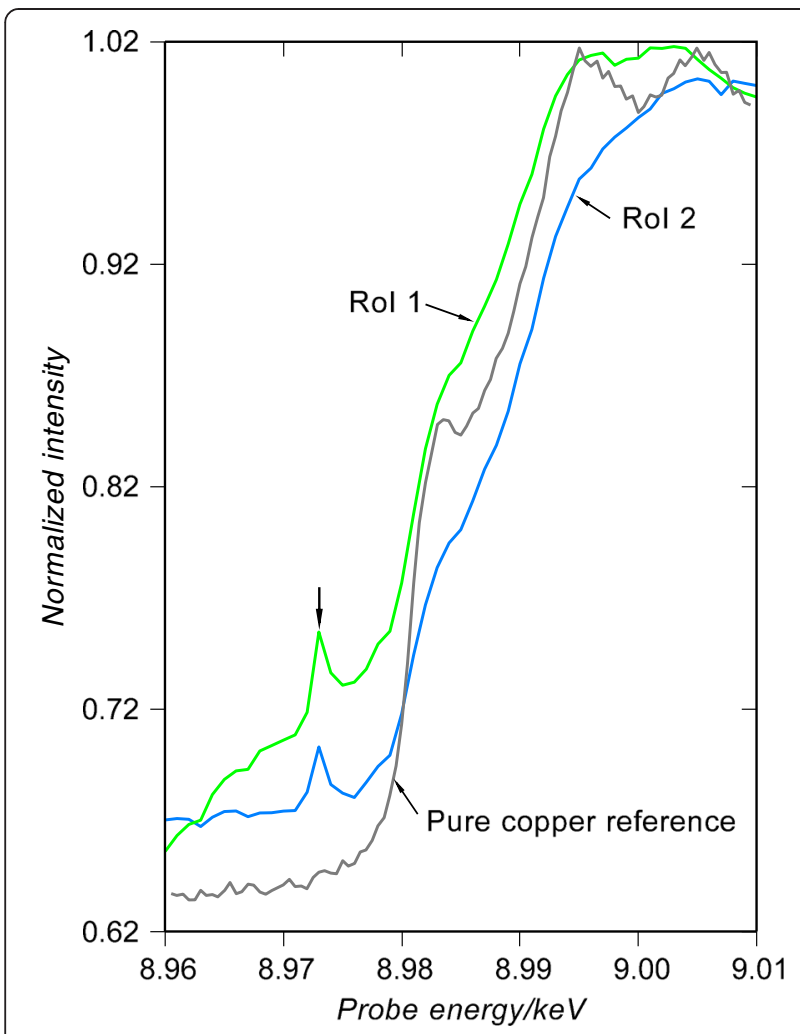

Figure $4 \mathrm{CU}$-K edge XEOL-XANES spectra from Rols shown in Figure $\mathbf{3}(\mathbf{b})$. Guided by the oxidation state image in Figure 3(c) regions of interest were set up on predominantly $\mathrm{Cu}(\mathrm{I})$ and $\mathrm{Cu}(\mathrm{II})$ areas. The Rols are shown in Figure 3(b) and respectively numbered 1 and 2. Rol 1 comprises two separate $\mathrm{Cu}(\mathrm{l})$ areas. The spectra are shown with one from a pure copper reference at the same monochromator calibration. The data from both Rols contains a shoulder at $8.983 \mathrm{keV}$ which is probably characteristic of the underlying copper. Otherwise The green curve from Rol 1 is consistent with cuprite XANES, whereas that from Rol 2 (blue curve) shows a shift characteristic of tenorite. The presence of copper-related features suggests that the patina is $<200 \mathrm{~nm}$ thick, or discontinuous. The reason for the arrowed pre-edge spike is not known.

area of $2.3 \times 2.3 \mathrm{~mm}^{2}$. This device was used to obtain the data in Figure 2.

The camera may be replaced by a broad band photomultiplier tube, Hamamatsu H8259-01 (Hamamatsu Photonics K. K., Japan) for filtered total ODXAS. This device can also be mounted on the sample manifold in parallel with an $\mathrm{X}$-ray fluorescence detector to give total ODXAS (no optical filter) and total XAS channels simultaneously with image acquisition. An optical spectroscopy system with parallel detection is under design for the microscope.

\section{The optical system}

At present the optics are made from fused silica which has a flat transmission above $93 \%$ per lens [27] across the required wavelength range $(250-1000 \mathrm{~nm})$, and virtually no visible fluorescence, even when struck by $x$-rays. The transmission is limited by reflection at the lens surfaces and not by transit through the fused silica. There are no coatings on the lenses in order to avoid secondary fluorescence. Since only one refractive index is available the system is not achromatic and for best image quality the optical bandwidth must be limited by filters. This is not normally a disadvantage because improved chemical speciation can result from differences in the colour spectrum naturally emitted by different compounds. As shown in Figure 6, the first lens is $50 \mathrm{~mm}$ from the sample to allow a large access volume, and reduce the backscattered $x$-ray flux at its surface. This large working distance also gives the device a large depth of focus - around $1 \mathrm{~mm}$ - so that rough or curved objects may be imaged. The first two lenses are custom planoconvex aspheres and form a compound objective with low geometrical aberration. This is followed by a bi-concave lens which is mounted in a motorised housing and used as the focussing element. Finally, a weak bi-convex lens is used to project the image onto the sensor surface. The overall magnification is 12 , so that a sensor pixel size of $\sim 12 \mu \mathrm{m}$ square, maps onto a $\sim 1 \mu \mathrm{m}$ square (virtual pixel) at the sample. This determines the ultimate lateral resolution of the system. The sensor is protected by a fused silica flat, so the 10 fused silica surfaces give an overall transmission of 70\%. A housing for $\varnothing 25 \mathrm{~mm}$ filters follows the projector lens. The filters used in this work were red and blue Techspec ${ }^{\circ}$ fluorescence line filters (Edmund Optics Ltd., UK) with pass bands of 612-644 nm (\# 84-103) and 457-487 nm (\#67-027) respectively (measured transmission $\geq 95 \%$ across the pass band, $<0.1 \%$ elsewhere [16]).

\section{Instrument control, acquisition and data processing}

XEOM 1 is controlled remotely using custom software esaXAS [16] running on a laptop PC which drives a pair of Agilent U2300A USB multifunction cards which provide control functions for the optics and sample handling. The software also controls the cameras using the relevant software functions. The software allows the user to set up a large variety of acquisition scenarios, automatically acquires and stores the image stacks and synchronizes image acquisition with the stepping of the monochromator on the synchrotron beam line.

Like the control software, the data processing software is custom written and forms part of the esaProject code (๔2006-2014 EVA Surface Analysis) [28].

\section{XEOL-XAS acquisition}

The data were acquired on beam line BM 28 (XMaS) at the ESRF. X-rays were incident at $60^{\circ}$ to the sample 


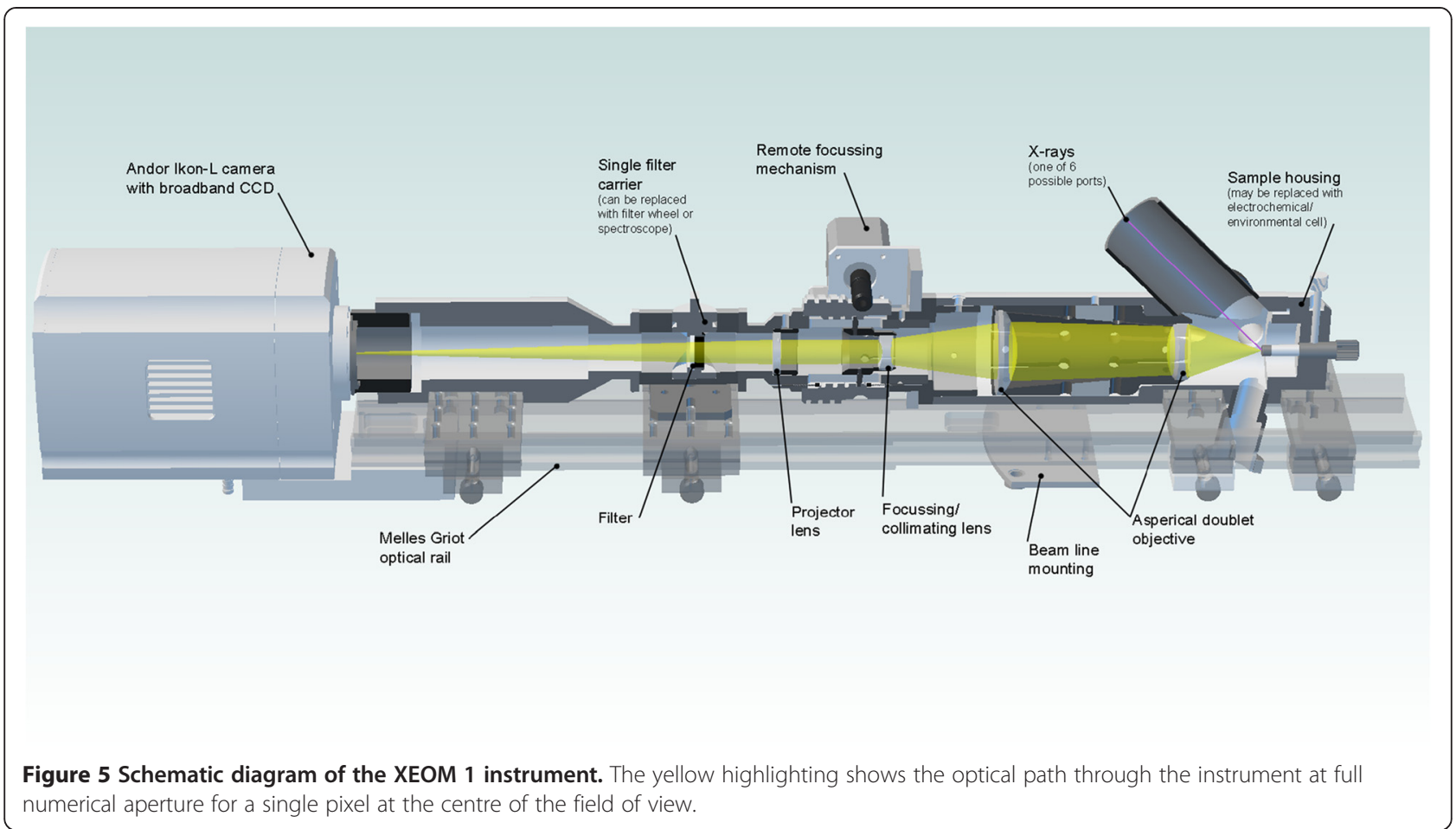

surface in a footprint around $1 \times 2 \mathrm{~mm}^{2}$ containing $10^{11}$ $10^{12}$ photons s $\mathrm{s}^{-1}$. At $9 \mathrm{keV}$ this corresponds to an input power density $<7.2 \times 10^{-3} \mathrm{~W} \mathrm{~mm}^{-2}$. Image stacks containing 51 to 112 images were acquired at uniform intervals across $x$-ray energy range $8.96-9.01 \mathrm{keV}$, thus spanning the $\mathrm{Cu}-\mathrm{K}$ absorption edge. Image acquisition times vary between 20 and 300 seconds in this work, but are constant for a given stack acquisition. In a typical experiment on a new material system, image stacks or total XEOL-XANES data (substituting a photomultiplier tube for the camera) are taken through a range of narrow band filters across the bandwidth of the device. Along with similar measurements on reference materials, this allows us to decide which filters to use in future analyses of similar samples.

\section{Test samples}

Synthetic nantokite was made on the surface of copper electron microscope grids $20 \mu \mathrm{m}$ thick and $\varnothing 3.05 \mathrm{~mm}$ with a pitch of $127 \mu \mathrm{m}$ (Athene Old 200, Agar Scientific Ltd. UK). The grid has bars $35 \mu \mathrm{m}$ wide and holes $90 \mu \mathrm{m}$ square. Cleaning these is impractical so they were used directly from the packaging. They were immersed for $1 \mathrm{~h}$ in a saturated aqueous solution of copper II chloride (Aldrich, >99\%) and then rinsed very briefly with deionized water to remove residual $\mathrm{CuCl}_{2}$. (This must be done rapidly or much of the $\mathrm{CuCl}$ patina will hydrolyse to $\mathrm{Cu}_{2} \mathrm{O}$ [28]). Cuprite was produced on $\varnothing 12.5 \mathrm{~mm} \times$ $2 \mathrm{~mm}$ thick coupons of $99.9 \%$ pure copper (Goodfellow, UK). The copper surface was cleaned as described elsewhere [28] and heated to a dull red in a reducing Bunsen

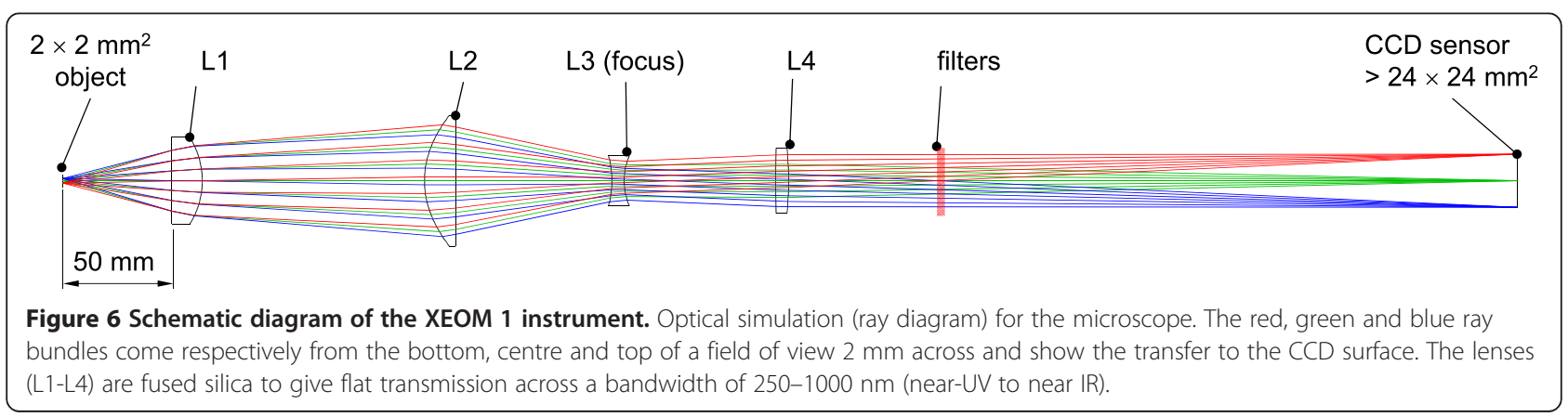


flame. A patina of $>90 \%$ cuprite with a residue of tenorite forms immediately on air exposure [29].

\section{Competing interests}

The authors declare that they have no competing interests.

\section{Authors' contributions}

AA and MGD conceived and managed the XEOM project which was supported by funding acquired by AA. MGD drafted the content of this paper using data obtained by all the authors over two beam time allocations. MH and MGD designed the hardware and wrote the software. $\mathrm{MH}$ designed the lenses. P-JS devised and made the reference samples. PT was the contact scientist on the beam line and made it all sing for us. All authors read and approved the final manuscript.

\section{Acknowledgements}

The XEOM 1 project is greatly indebted to numerous organizations and individuals viz: The Research Foundation-Flanders (FWO) and the Paul Instrument Fund of the Royal Society for the funding which supported the construction; EVA Surface Analysis (UK) which supplies the XEOM instrument engineering and the data processing software (esaProject); the beam-line team on BM28 (XMAS) at the ESRF, especially Simon Brown and Laurence Bouchenoire; Adrian Lovejoy, David Greenshields, and Bob Day of the Electronics Workshop at the University of Warwick who built the electronics; The Computing Group at the ESRF for advice on interfacing to ESRF systems; Nigel Poolton, Manolis Pantos and Rob Pettifer for their early help and guidance. MH and P-JS are grateful to the UK Engineering and Physical Sciences Research Council (EPSRC) and Ghent University respectively for their PhD studentships. XMaS is funded as a Mid-Range Facility by the EPSRC. Finally, the author's thanks go to the Trustees of the Mary Rose and Professor Mark Jones $\mathrm{MBE}$, Head of Collections, for allowing them access to material from this historic ship and to Rosie Grayburn, PhD student, who took care of the samples on the beam line.

\section{Author details}

${ }^{1}$ Department of Physics, University of Warwick, Coventry CV4 7AL, UK. ${ }^{2}$ Matt Hand, Diamond Light Source Ltd., Harwell Science and Innovation Campus, Didcot, Oxfordshire OX11 ODE, UK. ${ }^{3}$ Department of Analytical Chemistry, Ghent University, Ghent B9000, Belgium. ${ }^{4}$ XMaS The UK CRG, ESRF-The European Synchrotron, CS4022038043 Grenoble Cedex 9, France.

\section{Received: 13 October 2014 Accepted: 26 February 2015}

Published online: 01 May 2015

\section{References}

1. D'Silver A, Fassel V. Determination of uranium in ores by an $x$-ray excited optical luminescence technique. Anal Cherm. 1977:49:638-41.

2. Takashima K, Nakajima T, Okashita H, Shimizu R. Construction and performance of a newly developed X-ray excited optical luminescence spectrometer. Spectrochim Acta. 1981;162:687-95.

3. Hayakawa S, Hirose T, Yan L, Morishita M, Kuwano H, Gohsho Y. Synchrotron radiation excited optical luminescence for chemical state selective analysis. X-Ray Spectrom. 1999;28:515-8.

4. Kim P, Brandys M, Hu Y, Puddephatt J, Sham T-K. Soft X-ray excited optical luminescence studies of gold(I) complex with diphosphene and 4,4'-bipyridyl ligands. J Luminescence. 2003;105:21-6.

5. Soderholm L, Liu G, Antinio M, Lytl F. X-ray excited optical luminescence (XEOL) detection of $x$-ray absorption fine structure (EXAFS). J Chem Phys. 1998;109:6745-52.

6. Quinn F, Poolton N, Malins A, Pantos E, Andersen C, Denby P, et al. The mobile Luminescence End-Station, MOLES: a new public facility at Daresbury Synchrotron. J Synchrotron Rad. 2003;10:461-6.

7. Martínez-Criado G, Alén B, Sans J, Homs A, Kieffer I, Tucoulou R, et al. Spatially resolved $x$-ray excited optical luminescence. Nucl Instrum Meth Phys Res B. 2012;284:36-9.

8. Dowsett M, Adriaens A, Jones K, Poolton N, Fiddy S, Nikitenko S. Optically detected $\mathrm{x}$-ray absorption spectroscopy measurements as a means of monitoring corrosion layers on copper. Anal Chem. 2008;80:8717-24.

9. Sham TK, Gordon RA. RIXS, XEOL and XEOL Imaging of Rare-earth Phosphors at the L-edge. AIP Conference Proceedings. 2010;1234:133-6.
10. Adriaens A, Quinn P, Nikitenko S, Dowsett M. Real time observation of $x$-ray induced surface modification using simultaneous XANES and XEOL-XANES. Anal Chem. 2013;85:9556-63.

11. Schroeder SLM. Towards a 'universal curve' for total electron-yield XAS. Solid State Comm. 1996;98:405-9.

12. Taylor R, Finch A, Mosselmans J, Quinn P. The development of a XEOL and TR XEOL detection system for the 118 microfocus beamline Diamond light source. J Luminescence. 2013;134:49-58.

13. Dalby KN, Anderson AJ, Mariano AN, Gordon RA, Mayanovic RA, Wirth R. An investigation of cathodoluminescence in albite from the A-type Georgeville granite. Nova Scotia Lithos. 2010;114:86-94.

14. Thoury M, Echard J-P, Réfrégiers M, Berrie B, Nevin A, Jamme F, et al. Synchrotron UV-visible multispectral luminescence microscopy of historical samples. Anal Chem. 2011;83:1737-45.

15. Poolton N, Towlson B, Hamilton B, Evans D. New instrumentation for micro-imaging $x$-ray absorption spectroscopy using optical detection methods. Nucl Instrum Meth Phys Res B. 2006:246:445-51.

16. Hand M. Development of an $x$-ray excited optical luminescence microscope (XEOM). PhD Thesis. The University of Warwick; 2014.

17. Rosenberg RA, Zohar S, Keavney D, Divan R, Rosenmann D, Mascarenhas A, et al. Elemental and magnetic sensitive imaging using $x$-ray excited luminescence microscopy. Rev Sci Instrum. 2012;83:073701.

18. Verri G. The spatially resolved characterisation of Egyptian blue, Han blue and Han purple by photo-induced luminescence digital imaging. Anal Bioanal Chem. 2009;394:1011-21.

19. Scharf O, Ihle S, Ordavo I, Arkadiev V, Bjeoumikhov A, Bjeoumikhova S, et al. Compact pnCCD-Based X-ray Camera with High Spatial and Resolution: A Color X-ray Camera. Anal Chem. 2011;83:2532-8.

20. Tack P, Garrevoet J, Bauters S, Vekemans B, Laforce B, Van Ranst E, et al. Full-Field Fluorescence Mode Micro-XANES Imaging Using a Unique Energy Dispersive CCD Detector. Anal Chem. 2014;86:8791-7.

21. Larcheri S, Rocca F, Jandard F, Pailharey D, Graziola R, Kuzmin A, et al. $X$-ray excited optical luminescence detection by scanning near-field optical microscope: a new tool for nanoscience. Rev Sci Instrum. 2008;79:013702-1-9.

22. Chabala J, Levi-Seti R, Wang Y. Practical resolution limits of imaging microanalysis with a scanning ion microprobe. Appl Surf Sci. 1988;32:10-32

23. Rigaut J, Vassy J. High resolution three-dimensional images from confocal scanning laser microscopy quantitative study and mathematical correction of the effects from bleaching and fluorescence attenuation with depth. Anal Quant Cytol Histol. 1991;13:223-32.

24. Jefimovs K, Vila-Comamala J, Stampanoni M, Kaulich B, David C. Beam-shaping condenser lenses for full field transmission X-ray microscopy. J Synchrotron Rad. 2008;15:106-8.

25. Poolton N, Towlson B, Hamilton B, Wallinga J, Lang A. Micro-imaging synchrotron-laser interactions in wide band-gap luminescent materials. J Phys D Appl Phys. 2007:40:3557-62.

26. Dowsett $M$, Adriaens A. Cell for simultaneous synchrotron radiation $x$-ray and electrochemical corrosion measurements on cultural heritage metals and other materials. Anal Chem. 2006;78:3360-5.

27. Schott optical glass pocket catalogue [http://www.schott.com/ advanced_optics/english/download/schott-optical-glass-pocket-catalogeurope-october-2011-eng.pdf]

28. Dowsett M, Adriaens A, Martin C, Bouchenoire L. The use of synchrotron X-rays to observe copper corrosion in real time. Anal Chem. 2012;84:4866-72.

29. Sabbe P-J, Dowsett M, Hand M, Grayburn R, Thompson P, Bras W, et al. Evaluation of a X-ray-excited optical microscope (XEOM) for the chemical imaging of metal and other surfaces. Anal Chem. 2014;86:11789-96. 